\title{
Der Unterricht im Gegenstand Kulturpflege
}

\section{Sein Beitrag zur Schulentwicklung im Wertedialog und interkulturellen Lernen}

DOI: https://doi.org/10.53349/sv.2021.i2.a88

\begin{abstract}
Die private Berufsschule der SPAR AG in Wien bereichert seit 1958 mit Kulturpflege als eigenem Gegenstand die Schulkultur und Schulentwicklung durch Wertedialog, interkulturelles und interreligiöses Lernen. Die dabei im Begegnungslernen erworbenen Kompetenzen unterstützen das Miteinanderleben, -lernen und -arbeiten der Lehrlinge und befruchten die praktische Ausbildung in den Filialen im Umgang mit den Mitarbeitenden und der Kundschaft. Die wertschätzende und anerkennende Wahrnehmung von Diversität und Differenz ist der Motor des Gegenstandes, der kontextuell in alle fächerübergreifenden Projekte eingebunden ist.
\end{abstract}

Kulturpflege, Wertedialog, interkulturelles Lernen, Begegnungslernen

\section{Die private Berufsschule der SPAR AG}

SPAR Österreich ist weltweit das einzige Handelsunternehmen, das als Schulerhalter eine eigene Berufsschule als Privatschule mit Öffentlichkeitsrecht betreibt. Gegründet wurde die Schule 1906 durch Julius Meinl II., der als Sohn des Gründers des damals im österreichischen Lebensmittelhandel aufstrebenden Unternehmens die Qualität in seinen Geschäften steigern wollte und dafür gut ausgebildete Verkaufskräfte benötigte. Es galt auch die geringe Wertschätzung für Lehrlinge im Lebensmittelhandel zu verbessern und die jungen Mitarbeitenden, die im damaligen Jargon als „Budelhupfer“ bezeichnet wurden, auf eine höhere soziale Stufe zu stellen. Die für Lehrlinge damals übliche Schulbildung (Abend- oder Sonntagsschulen mit bis zu fünfzig Lehrlingen pro Klasse) schien ihm für die Umsetzung seiner Ziele nicht geeignet. So gründete er 1906 mit vierzehn Lehrlingen eine eigene Kaufmännische Fortbildungsschule, da er vierzehn Einzelhandelsgeschäfte in Wien führte und in jedem Geschäft 
auch Jugendliche ausbilden wollte. Am 24. November 1906 genehmigte der k.k. niederösterreichische Landesschulrat per Dekret die Errichtung und Führung der Schule und verlieh das Öffentlichkeitsrecht.

Als die SPAR AG im Jahr 2000 die Meinl-Filialen gekauft und übernommen hatte, fiel auch die Entscheidung, die private Berufsschule nicht nur weiterzuführen, sondern die Zahl der Lehrlinge in Wien massiv zu erhöhen - SPAR ist österreichweit der größte Lehrlingsausbildner und den Schulstandort aus- und umzubauen. Ab dem Schuljahr 2000/01 führt die SPAR AG mit staatlicher Genehmigung als Schulerhalter die „private Berufsschule der SPAR AG mit Öffentlichkeitsrecht". Im Schuljahr 2020/21 wurden achtzehn Klassen eröffnet, in denen rund 390 Lehrlinge im Lehrberuf „Einzelhandel Schwerpunkt Lebensmittel und Feinkostfachverkauf" unterrichtet wurden.

\section{Der Gegenstand „Kulturpflege“}

Im Jahre 1958, also noch vor der gesetzlichen Einführung des Freigegenstandes Religion an Berufsschulen 1962, wurde dieser Gegenstand an unserer Berufsschule entwickelt. In seiner Durchführung erhalten die Jugendlichen die Möglichkeit, sich mit Bildungsinhalten auseinanderzusetzen, die mit Lebensgestaltung, Persönlichkeitsentwicklung, Wertordnung(en), Wertorientierung, Kultur, Gesellschaftsentwicklung, Brauchtum, zwischenmenschlichen Beziehungen und der sogenannten Sinnsuche im Leben zu tun haben.

Der für alle Lehrlinge verbindliche Gegenstand wird in allen drei Jahrgängen mit je einer Wochenstunde geführt. Er wird nicht mit Noten klassifiziert, in Schulnachrichten und Jahreszeugnissen wird die Teilnahme aber bestätigt. Der Schulerhalter erstellt die Lehrpläne und Lehrstoffverteilungen in Absprache und Kooperation mit dem Amt für Schule und Bildung der Erzdiözese Wien. Jenes Amt vermittelt für diesen Gegenstand geeignete und qualifizierte Lehrende, die durch die zuständige Fachinspektion begleitet werden.

\section{Wertedialog, interkulturelles und interreligiöses Lernen und Schulentwcklung}

Im Schuljahr 2020/21 setzte sich unsere Schulgemeinschaft mit Lehrlingen aus 31 verschiedenen Nationen und Volksgruppen zusammen, die 33 Sprachen beherrschten und sechzehn Religionsbekenntnissen angehörten. Mit dieser multikulturellen und multireligiösen Zusammensetzung unserer Schulgemeinschaft - ein „Spiegel“ unserer gesellschaftlichen Realität, auch die der Mitarbeitenden in den einzelnen SPAR-Filialen und der Zusammensetzung unserer Kundschaft bei der Begegnung im Verkauf - stehen uns eine Vielfalt von Meinungen, Ideen, Erfahrungen und Lebensstilen zur Verfügung.

Die Diversität kann nicht ignoriert werden, sondern soll als Normalität zu uns gehören. Dafür braucht es aber Methoden, Raum und Zeit, um die Pluralität als Wert an sich zu erkennen 
und die gemeinsamen, tragenden Werte als Basis für unser gemeinsames Lernen, Arbeiten und Leben wie eine "Schnittmenge" in der Vielfalt herauszuarbeiten. Andererseits ist aber auch die Differenz zu benennen und mit gegenseitiger Anerkennung und Wertschätzung zu versehen. Diese Sicht lässt die interkulturelle Bildungsdimension in der Schule so spannend sein, wenn Reflexion und Austausch überfließen in wertschätzende Begegnungen, in gemeinsames Feiern, in das gelebte Zeugnis von Werten, die der ganzen Schulgemeinschaft zu einem gelingenden Miteinander gereichen.

Gerade auch die religiöse Dimension von Bildung kann hierbei nicht ignoriert (also privatisiert) oder ideologisiert werden (indem Differenz und Vielfältigkeit übersehen werden), sondern sie ist in Diversität ein dynamischer Lernprozess für gegenseitige Wahrnehmung und Wertschätzung. Diese Dynamik schließt ein, dass die Kommunikations-, Frage- und Ausdrucksfähigkeit im Feld der interkulturellen und interreligiösen Bildung erweitert wird und die Lehrlinge in der Lage sind, ihren eigenen, persönlichen Standpunkt zu artikulieren und den der anderen zu verstehen. Neben den inhaltlichen Kompetenzen knüpfen wir an die ganzheitliche Dimension von kultureller Bildung, Religion und Sinnsuche an, die Körper, Seele und Geist umfasst und sich auch in alternativen Methoden und vielfältigen Lernorten widerspiegelt (z.B. Räume und Zeiten der Stille und Besinnung, ästhetisches Lernen in der Sakralraumpädagogik, multikulturelle und multireligiöse Feiern, Körpersprache und Gesten in den verschiedenen Kulturen, ...).

Der Kulturpflegeunterricht ist im Sinne eines kontextuellen Lernens fächerübergreifend in alle Zusatzausbildungen unserer Lehrlinge integriert und beteiligt. So werden alle Lehrlinge des ersten Jahrganges in Kooperation mit FAIRTRADE Österreich zu „FAIRTRADE-Botschafterinnen und Botschaftern" ausgebildet. In Kulturpflege beschäftigen wir uns daher mit den Themenfeldern "Solidarität - in meinem Umfeld und in der Welt ", "Gerechtigkeit", „,die goldene Regel in den Weltreligionen als Motor für gelingendes Leben in Gemeinschaft" und "mein konkretes Engagement für eine gerechte Welt“. Die dritten Jahrgänge werden in Zusammenarbeit mit dem WWF zu „Green Champions" ausgebildet und beschäftigen sich mit dem Weg in eine nachhaltige Zukunft. Hierbei behandeln wir Themen wie „Leben als Gabe und Aufgabe", „Umweltethik“, "die Bedeutung von Essen und Trinken in den Weltreligionen“ und reflektieren die konkreten Möglichkeiten von nachhaltigen Alternativen im privaten, aber auch im beruflichen Umfeld in den SPAR-Filialen.

Im Konzept des Kulturpflegeunterrichtes ist immer zu berücksichtigen, dass die erworbenen interkulturellen und interreligiösen Kompetenzen nicht nur in der Berufsschule angewendet werden können, sondern auch im Familien- und Freundeskreis und natürlich als wichtige Qualifikationen im Einzelhandel im Umgang mit den anderen Mitarbeitenden und der Kundschaft.

Begegnungslernen und Dialog im miteinander und voneinander Lernen sind für das Konzept des Kulturpflegeunterrichtes der „Königsweg“. In der Wahrnehmung von verschiedenen kulturellen und religiösen Zeugnissen ist die Differenzerfahrung der Motor des Nachdenkens und lässt in existenzielle Auseinandersetzung geraten, eigene Standpunkte finden und eigene 
Erfahrungen machen. Die erworbenen interkulturellen und interreligiösen Kompetenzen in den beschriebenen Kommunikations- und Handlungsfähigkeiten werden im Idealfall in das weitere persönliche Leben der Lehrlinge integriert und weiter kreativ entfaltet werden. Für die Berufsschulzeit ist die Ausbildung dieser Kompetenzen allemal ein wesentlicher Beitrag zu Schulkultur und Schulentwicklung.

Das Lernkonzept Kulturpflegeunterricht, das natürlich einer permanenten Evaluierung und Weiterentwicklung bedarf, ist ein eindeutiges Markenzeichen unserer Berufsschule und wird von unserem Schulerhalter SPAR auch in der Werbung eingesetzt (so zum Beispiel in dem Slogan „SPAR setzt Zeichen ... für gelebte Integration!“).

Stolz sind wir in diesem Zusammenhang auch auf die Verleihung des „Österreichischen Integrationspreises" für dieses Lernkonzept.

\section{Autor}

Horst Pater Nikolaus Schachtner, MMag.

Pädagogischer Leiter der Berufsschule der SPAR AG, Augustinermönch

Kontakt: horst.schachtner@spar-akademie.at 